\title{
Repellency, toxicity, and oviposition inhibition of vegetable extracts against greenhouse whitefly Trialeurodes vaporariorum (Westwood) (Hemiptera: Aleyrodidae)
}

\author{
Edgar Eduardo Mendoza-García ${ }^{1 *}$, Laura Delia Ortega-Arenas', Rafael Pérez-Pacheco², \\ and Cesáreo Rodríguez-Hernández ${ }^{1}$
}

\begin{abstract}
In a search for sustainable options of greenhouse whitefly Trialeurodes vaporariorum (Westwood) management, the toxic and/or repellent potential of water, ethanolic, and acetonic extracts of Ambrosia artemisiifolia L. (Asteraceae), Comocladia engleriana Loes (Anacardiaceae), Piper auritum Kunth (Piperaceae), Raphanus raphanistrum L. (Brassicaceae), and Taraxacum officinale F.H. Wigg. aggr.* (Asteraceae) were evaluated. Repellency was assessed by the cylinder method (olfactometer), while toxicity and oviposition inhibition were assessed by the leaf immersion method. Acetonic extracts did not cause any repellent or insecticidal effect. In contrast, $200 \mathrm{mg} \mathrm{mL}^{-1}$ water and ethanolic extracts of $R$. raphanistrum and ethanolic extract of $A$. artemisiifolia had the highest repellent activity (76\%, 72\%, and 69\%, respectively) although their activity decreased gradually over time. Ethanolic extracts of P. auritum (66\%) and R. raphanistrum $(56 \%)$ at $200 \mathrm{mg} \mathrm{mL}^{-1}$ were highlighted as being toxic, while the most effective in inhibiting oviposition were water extracts of $R$. raphanistrum (76.1\%) and P. auritum (72.0\%) and ethanolic extract of P. auritum (69.5\%); however, concentrations lower than $60 \mathrm{mg}$ $\mathrm{mL}^{-1}$ caused oviposition stimulation. Our results suggest that water and ethanolic extracts of $R$. raphanistrum and $P$. auritum represent a useful tool in integrated whitefly management.
\end{abstract}

Key words: Botanical insecticides, IPM, Trialeurodes vaporariorum.

\section{INTRODUCTION}

The greenhouse whitefly Trialeurodes vaporariorum (Westwood) (Hemiptera: Aleyrodidae) is a major worldwide pest of vegetable and ornamental crops. It causes direct damage and transmits viruses that cause serious diseases (Jones, 2003). To maintain whiteflies under control, farmers use broad-spectrum insecticides; they also apply higher rates and mixtures, which result in the development of resistant populations (Ortega Arenas et al., 1998; Ortega, 2008), increase production costs, elevate environmental pollution, and cause health problems in farmers and consumers (Agnihotri, 1999; Rodríguez and Vendramim, 2008). This situation has motivated the generation and implementation of alternative or complementary control strategies, such as the use of insectistatic and insecticidal plants for pest

${ }^{1}$ Colegio de Postgraduados, Campus Montecillo, km 36.5 Carr. México-Texcoco, C.P. 56230, Montecillo, México.

"Corresponding author (mendoza.edgar@colpos.mx).

${ }^{2}$ Instituto Politécnico Nacional, Centro Interdisciplinario de Investigación para el Desarrollo Integral Regional-Unidad Oaxaca, Calle Hornos 1003, Col. Indeco Xoxocotlán C.P. 71230, Oaxaca, México.

Received: 11 July 2013.

Accepted: 18 December 2013.

doi: $10.4067 /$ S0718-58392014000100007 management without risk to human or environmental health (Rodríguez, 2000).

It is estimated that there are more than 2000 plant species with insectistatic and insecticidal effects that could be included in an integrated pest management program for whiteflies (Grainge and Ahmed, 1988; Rodríguez, 1998; 2000; Rodríguez and Vendramim, 2008). Among the most used plants are onion Allium cepa L., garlic A. sativum L. (Amaryllidaceae), neem Azadirachta indica A. Juss. (Meliaceae), chili pepper Capsicum annuum L., tobacco Nicotiana tabacum L. (Solanaceae), castor-bean Ricinus communis L. (Euphorbiaceae), marigold Tagetes erecta L., and Irish lace T. filifolia Lag. (Asteraceae) (Grainge and Ahmed, 1988; Rodríguez and Vendramim, 2008; Camarillo et al., 2009). Previous results show that products obtained from these plants, released through natural sources or extracted and sprayed on crops, control the greenhouse whitefly by killing eggs, nymphs, and adults; they also permit population management, crop foliage protection, population reduction, adult repellency, and feeding, oviposition, and growth inhibition, or virus transmission reduction. Moreover, they have low toxicity for mammals and little impact on natural enemies; they are compatible with other strategies used in integrated pest management, are available and cheaper than conventional insecticides (Rodríguez, 1998; Rodríguez and Vendramim, 2008). Although plant diversity in Mexico 
is high, the potential of whitefly species management has not been explored. There are several papers that indicate that Mexican pepperleaf (Piper auritum Kunth, Piperaceae) is rich in secondary metabolites that have shown anti-feeding, fungicidal, bactericidal, cytotoxic, stimulating insecticidal, and synergistic activity (Delgado and Cuca, 2007; Scott et al., 2008); these leaves and inflorescence extracts of $P$. auritum are essentially a mixture of alkaloids, safrol, amines, butenolides, flavonoids, terpenes, among others compounds, which suggest potential insecticidal activity (Parmar et al., 1997; García et al., 2007; Sánchez et al., 2009). It is also known that common ragweed (Ambrosia artemisiifolia L.: Asteraceae) (Altieri, 1995), "titatil" (Comocladia engleriana Loes: Anacardiaceae), wild radish (Raphanus raphanistrum L.: Brassicaceae) (Jbilou et al., 2006; McCutcheon et al., 2009), and dandelion (Taraxacum officinale F.H. Wigg. aggr.*: Asteraceae) (Jovanović et al., 2007; Morar et al., 2008) are species that have proven to be effective to control different pests; however, in the reviewed literature there is no information about the use of plant extracts against whiteflies. This study was carried out to evaluate the biological activity of water, ethanolic, and acetonic extracts of $A$. artemisiifolia, $C$. engleriana, T. officinale, $P$. auritum, and $R$. raphanistrum against the greenhouse whitefly $T$. vaporariorum.

\section{MATERIALS AND METHODS}

The study was conducted in a greenhouse in the area of vector insects at the Colegio de Postgraduados, Montecillo, in Texcoco, Mexico, from September 2009 to November 2010.

\section{Trialeurodes vaporariorum strain}

The $T$. vaporariorum strain was established with approximately 2000 adults (2:1) from a colony isolated under greenhouse conditions since 2002. Adults were introduced into entomological cages $(60 \times 40 \times 60 \mathrm{~cm})$ covered with fine mesh in which 30-d-old 'Bayo Mex' bean plants (Phaseolus vulgaris L.) were maintained in plastic pots containing a mixture of vermicompost, leaf soil, and vermiculite $(3: 2: 1)$ as support medium. The adults were maintained on these plants for $1 \mathrm{wk}$ and then removed with a manual vacuum. Infested plants were transferred to another cage and maintained under greenhouse conditions (25 $\pm 5^{\circ} \mathrm{C}$ and $12: 12 \mathrm{~h}$ photoperiod) until the emergence of new adults. This process was carried out periodically to provide biological material for experiments. To confirm species identity, some specimens were identified using Martin (1987) and Martin and Mound (2007) keys.

\section{Plant collection and extract preparation}

Ambrosia artemisiifolia plants were purchased in local markets in the city of Oaxaca and $C$. engleriana plants in the community of Bajos de Chila in Mixtepec, Oaxaca,
Mexico. Piper auritum plants were collected from Zaachila county, $R$. raphanistrum $5 \mathrm{~km}$ from Tlacotepec Plumas county, and $T$. officinale from the Instituto Tecnológico del Valle de Oaxaca in Nazareno Xoxocotlán county, Oaxaca, Mexico. In all cases, plants were selected only if they were abundant and at the flowering stage. Species were identified by MSc María de los Remedios Aguilar Santelises, herbarium curator of the Centro Interdisciplinario de Investigación para el Desarrollo Integral Regional del Instituto Politecnico Nacional (CIIDIR-IPN) in the Oaxaca unit using Calderón and Rzedowski's (2001) taxonomic keys and comparing specimens with those existing in the herbarium.

The plants (approximately $50 \mathrm{~kg}$ each) were dried in the shade at room temperature $\left(25 \pm 2{ }^{\circ} \mathrm{C}\right)$ for $7 \mathrm{~d}$. The aerial parts were then pulverized in a stainless steel mill; the resulting powder was sieved with a 20 mesh sieve (0.86 mm opening).

Water extracts were prepared $1 \mathrm{~d}$ before their use to obtain the maximum water-soluble compounds, $80 \mathrm{~g}$ of powder were placed in a $250 \mathrm{~mL}$ polyethylene jar, and $200 \mathrm{~mL}$ distilled water were added. The mixture was shaken and let to rest for $24 \mathrm{~h}$ at room temperature $(25 \pm 2$ $\left.{ }^{\circ} \mathrm{C}\right)$. The solution was later filtered through a fine organdy cloth to obtain the liquid part $(20 \%)$.

To prepare the acetonic and ethanolic extracts, 100 $\mathrm{g}$ powder of the respective plants were placed in a 1 $\mathrm{L}$ jar and $500 \mathrm{~mL}$ of solvent (acetone or ethanol) were added. The mixtures were shaken and let to rest for 72 $\mathrm{h}$ at room temperature. Later, the solution was filtered and the solvent evaporated in a distillator $\left(78{ }^{\circ} \mathrm{C}\right.$ at 1 atm $70 \mathrm{rpm}$ and $55-60{ }^{\circ} \mathrm{C}$ at 1 atm $30 \mathrm{rpm}$ ) to obtain the crude extract. Extracts were stored in amber jars for 2 wk and refrigerated at $4{ }^{\circ} \mathrm{C}$. Products were resuspended in distilled water to prepare a $20 \%$ solution from which subsequent dilutions were performed.

To assess the effect of each concentration and replicate, 20 greenhouse whitefly adults of unknown sex and 3-5 d old were starved for $2 \mathrm{~h}$ before the test. Distilled water (control) was always included. All the treatments included a control and 1\% Tween 20 was added as an adherent before extract application. Five replicates were used for each concentration. First, response intervals were assessed for each extract by a preliminary experiment (biological response window) using concentrations from 20\% (200 $\left.\mathrm{mg} \mathrm{mL}^{-1}\right)$ to $0.00001 \%\left(0.0001 \mathrm{mg} \mathrm{mL}^{-1}\right)$ to detect insect mortality, oviposition inhibition, or repellency rates in the range of 0 to 100 . Only the treatments that caused significant activity ( $\geq 30 \%$ ) were evaluated again with logarithmic concentrations intercalated among those that had the activity to perform a complete test.

\section{Repellency bioassay}

Repellency was evaluated by the olfactometer method proposed by Schuster et al. (2009). This method consisted in submerging a leaf disk, $3 \mathrm{~cm}$ in diameter from 15 to 20- 
d- old 'Bayo Mex' bean plants in separate concentrations of the test solutions. After drying for approximately $20 \mathrm{~min}$, the disk with the exposed adaxial surface was held in a bag, which was pushed into a hole in the lid of each cylinder with the adaxial leaf surface facing into the cylinder. Twenty adults were introduced into each cylinder. Repellency was measured by the difference in the number of insects settled on the leaf disk control and the disk treated at $3,4,5,6$, and $24 \mathrm{~h}$ after introduction expressed as a percentage and considered the total number of adults as $100 \%$ in each replicate. Data were corrected with those obtained in the control by Abbott's equation (Abbott, 1925) and subjected to the Kruskal-Wallis test to calculate variances $(p \leq 0.05)$ with SAS Institute (1999). Probit analyses were also performed to predict the concentration of each extract that would reduce settling of adults by $50 \%\left(\mathrm{RC}_{50}\right)$ and were expressed in $\mathrm{mg} \mathrm{mL}^{-1}$. The Repellency Index (RI) proposed by Lin et al. (1990) was estimated to compare the response among treatments and calculated with the equation $R I=2 G /(G+P)$ where $\mathrm{G}$ is the percentage of insects in the treatment and $\mathrm{P}$ is the percentage of insects in the control. Values were classified as $\mathrm{RI}=1$ neutral plant, $\mathrm{RI}<1$ repellent plant, and $\mathrm{RI}>1$ attractive plant. As a safety margin for that classification, the value of the standard error for each treatment was added/subtracted.

\section{Adult mortality bioassay}

Mortality was evaluated by the leaf immersion method proposed by Ortega Arenas et al. (1998). A bean leaflet, 15-20 d old with extended lamina, was submerged in the test solution. After drying for approximately $20 \mathrm{~min}$, a 3 $\mathrm{cm}$ circular polyurethane clip-type entomological cage was attached to the treated leaflet. Twenty whitefly adults were introduced into the cage through a lateral hole with a small vacuum. Treated plants were maintained under the same environmental conditions in the rearing strain. After $24 \mathrm{~h}$, mortality was recorded with a stereoscopic microscope. A dead insect was one that was unable to move or change position when pressure was put on its abdomen with a dissecting needle. As in repellency bioassays, data were corrected with those obtained in the control and subjected to probit analysis (SAS Institute, 1999) to predict the concentration of each extract that would cause $50 \%\left(\mathrm{LC}_{50}\right)$ mortality of adults, which was expressed in $\mathrm{mg} \mathrm{mL}^{-1}$.

\section{Oviposition inhibition bioassay}

To determine oviposition inhibition, a bean leaflet 15-20 $\mathrm{d}$ old with extended lamina was used; it was submerged in the test solution, dried at environmental temperature, and then put in a circular polyurethane clip-type entomological cage $(3 \mathrm{~cm}$ ). Twenty whitefly adults (sex ratio $1: 1$ ) were introduced into the cage through a lateral hole with a small vacuum and after $24 \mathrm{~h}$ the number of oviposited eggs was determined. Oviposition inhibition was also expressed as a percentage by considering the number of eggs of the control as 100\% (Ortega et al., 1998). Data were corrected by Abbott's equation (1925) and subjected to probit analysis (SAS Institute, 1999) to determine the concentration of each extract that would cause inhibition of oviposition by $50 \%\left(\mathrm{IOC}_{50}\right)$, which was expressed in $\mathrm{mg} \mathrm{mL} \mathrm{L}^{-1}$.

\section{RESULTS AND DISCUSSION}

Trialeurodes vaporariorum adults showed differential susceptibility to the evaluated extracts $(\mathrm{P} \leq 0.05)$. In preliminary bioassays, acetonic and some water extracts did not cause toxic activity or significant repellency $(\geq$ $30 \%$ ); for this reason, they were excluded in later detailed tests in the investigation. In contrast, several water and ethanolic extracts caused significant biological activity, so they were subjected to detailed tests.

\section{Repellency of adults}

The repellent effect of extracts varied according to species, time, and concentration $(\mathrm{P} \leq 0.05)$. In general, ethanolic extracts were more effective than water and acetonic extracts and their effect was positively related to concentration. Water and ethanolic extracts of $R$. raphanistrum at $200 \mathrm{mg} \mathrm{mL}$-1 showed the strongest repellent activity $3 \mathrm{~h}$ after application $(72 \%$ and $76 \%$, respectively) although it diminished over time ( $\mathrm{P} \leq 0.05$; $\mathrm{X}^{2}=13.59$ and 32.13) (Tables 1 and 2). In the other treatments, the response was variable, but significant repellency ( $\geq 30 \%$ ) was obtained with water extracts of $A$. artemisiifolia, $R$. raphanistrum, and T. officinale applied at concentrations above $100 \mathrm{mg} \mathrm{mL}^{-1}$, ethanolic extracts of $A$. artemisiifolia, $R$. raphanistrum, and $T$. officinale at $60 \mathrm{mg} \mathrm{mL}^{-1}$, and ethanolic extract of $T$. officinale at $40 \mathrm{mg}$ $\mathrm{mL}^{-1}$ (Tables 1 and 2). The repellent effect was unstable over time in all treatments. This response was confirmed with the RI values of $0.88 \pm 0.09$ for water extracts of $R$. raphanistrum and $0.87 \pm 0.11$ for $T$. officinale extracts at $60 \mathrm{mg} \mathrm{mL}^{-1}$. A similar response was achieved with $A$. artemisiifolia extract at $135 \mathrm{mg} \mathrm{mL}^{-1}(\mathrm{RI}=0.82 \pm 0.13)$ $3 \mathrm{~h}$ after application. However, the repellent effect only persisted up to $4 \mathrm{~h}$ with $R$. raphanistrum and $T$. officinale ( $\mathrm{RI}=0.57 \pm 0.30$ and $0.81 \pm 0.11$, respectively) with 200 $\mathrm{mg} \mathrm{mL} \mathrm{m}^{-1}$ extracts (Tables 1 and 2).

The $\mathrm{RC}_{50}$ values oscillated between 154.3 and 195.5 $\mathrm{mg} \mathrm{mL} \mathrm{L}^{-1}$. Three hours after the application, ethanolic and water extracts of $R$. raphanistrum showed the highest repellent activity at the $\mathrm{RC}_{50}$ level $(154.3$ and $156.4 \mathrm{mg}$ $\mathrm{mL}^{-1}$, respectively); this was followed by ethanolic extract of A. artemisiifolia (187.5 mg mL $\mathrm{m}^{-1}$ ), water extract of $T$. officinale (190.9 $\mathrm{mg} \mathrm{mL}^{-1}$ ), ethanolic extract of P. auritum (192.1 $\mathrm{mg} \mathrm{mL}^{-1}$ ), and water extract of $R$. raphanistrum (195.5 $\left.\mathrm{mg} \mathrm{mL}^{-1}\right) 4 \mathrm{~h}$ after application (Tables 1 and 2). In general, the repellent activity of extracts decreased notably $4 \mathrm{~h}$ after being applied and practically failed at 
Table 1. Mean repellency $(\%)$ of adult whitefly Trialeurodes vaporariorum $3,4,5,6$, and $24 \mathrm{~h}$ after applying water extracts of three plant species.

\begin{tabular}{|c|c|c|c|c|c|c|c|c|c|c|}
\hline \multirow[b]{2}{*}{$\begin{array}{l}\text { Conc. } \\
(\mathrm{mg} \\
\left.\mathrm{mL}^{-1}\right)\end{array}$} & \multicolumn{4}{|c|}{$\begin{array}{c}\text { Ambrosia } \\
\text { artemisiifolia }\end{array}$} & \multicolumn{3}{|c|}{$\begin{array}{c}\text { Raphanus } \\
\text { raphanistrum }\end{array}$} & \multicolumn{3}{|c|}{$\begin{array}{l}\text { Taraxacum } \\
\text { officinale }\end{array}$} \\
\hline & Hour & $\begin{array}{l}\text { Repel. } \\
(\%)^{1}\end{array}$ & $\mathrm{RI}^{2}$ & $\mathrm{CL}^{3}$ & $\begin{array}{c}\text { Repel } \\
(\%)\end{array}$ & RI & CL & $\begin{array}{c}\text { Repel. } \\
(\%)\end{array}$ & RI & CL \\
\hline 200 & & 38 & $0.79 \pm 0.17$ & $\mathrm{R}$ & 72 & $0.46 \pm 0.32$ & $\mathrm{R}$ & 52 & $0.67 \pm 0.21$ & $\mathrm{R}$ \\
\hline 135 & & 34 & $0.82 \pm 0.13$ & $\mathrm{R}$ & 40 & $0.78 \pm 0.14$ & $\mathrm{R}$ & 47 & $0.72 \pm 0.23$ & $\mathrm{R}$ \\
\hline 100 & & 34 & $0.82 \pm 0.19$ & $\mathrm{~N}$ & 32 & $0.84 \pm 0.13$ & $\mathrm{R}$ & 41 & $0.77 \pm 0.19$ & $\mathrm{R}$ \\
\hline 60 & 3 & 22 & $0.90 \pm 0.15$ & $\mathrm{~N}$ & 27 & $0.88 \pm 0.09$ & $\mathrm{R}$ & 27 & $0.87 \pm 0.11$ & $\mathrm{R}$ \\
\hline 40 & & 23 & $0.89 \pm 0.29$ & $\mathrm{~N}$ & 22 & $0.91 \pm 0.18$ & $\mathrm{~N}$ & 23 & $0.89 \pm 0.24$ & $\mathrm{~N}$ \\
\hline 10 & & 19 & $0.92 \pm 0.13$ & $\mathrm{~N}$ & $11^{4}$ & $0.98 \pm 0.20$ & $\mathrm{~N}$ & 8 & $0.98 \pm 0.11$ & $\mathrm{~N}$ \\
\hline 3.5 & & 8 & $0.98 \pm 0.11$ & $\mathrm{~N}$ & $12^{4}$ & $0.97 \pm 0.23$ & $\mathrm{~N}$ & 7 & $0.99 \pm 0.09$ & $\mathrm{~N}$ \\
\hline Con & trol & 5 & - & - & 7 & - & - & 5 & - & - \\
\hline $\operatorname{Pr}>$ & $X^{2}$ & & 0.0003 & & & $<0.0001$ & & & $<0.0001$ & \\
\hline $\mathrm{X}^{2}$ & & & 27.31 & & & 32.13 & & & 33.19 & \\
\hline $\mathrm{RC}$ & & & - & & & $56.4(97.1-41$ & $110)^{5}$ & 190 & $9.9(150.4-26$ & 63.3) \\
\hline $\mathrm{b} \pm$ & & & - & & & $1.2 \pm 0.3$ & & & $1.3 \pm 0.1$ & \\
\hline 200 & & 23 & $0.90 \pm 0.17$ & $\mathrm{~N}$ & 610 & $0.57 \pm 0.30$ & $\mathrm{R}$ & 33 & $0.81 \pm 0.11$ & $\mathrm{R}$ \\
\hline 135 & & 23 & $0.90 \pm 0.13$ & $\mathrm{~N}$ & 28 & $0.84 \pm 0.23$ & $\mathrm{~N}$ & 29 & $0.84 \pm 0.16$ & $\mathrm{~N}$ \\
\hline 100 & & 19 & $0.93 \pm 0.11$ & $\mathrm{~N}$ & 22 & $0.88 \pm 0.18$ & $\mathrm{~N}$ & 25 & $0.87 \pm 0.25$ & $\mathrm{~N}$ \\
\hline 60 & 4 & 16 & $0.95 \pm 0.16$ & $\mathrm{~N}$ & 18 & $0.91 \pm 0.11$ & $\mathrm{~N}$ & 15 & $0.93 \pm 0.12$ & $\mathrm{~N}$ \\
\hline 40 & & 18 & $0.94 \pm 0.26$ & $\mathrm{~N}$ & 11 & $0.95 \pm 0.08$ & $\mathrm{~N}$ & 10 & $0.96 \pm 0.12$ & $\mathrm{~N}$ \\
\hline 10 & & 9 & $0.99 \pm 0.16$ & $\mathrm{~N}$ & 64 & $0.97 \pm 0.16$ & $\mathrm{~N}$ & 8 & $0.97 \pm 0.11$ & $\mathrm{~N}$ \\
\hline 3.5 & & 4 & $1.01 \pm 0.08$ & $\mathrm{~N}$ & 54 & $0.98 \pm 0.10$ & $\mathrm{~N}$ & 5 & $0.98 \pm 0.10$ & $\mathrm{~N}$ \\
\hline Con & trol & 3 & - & - & 1 & - & - & 2 & - & - \\
\hline $\operatorname{Pr}>$ & & & 0.0010 & & & $<0.0001$ & & & $<0.0001$ & \\
\hline $\mathrm{X}^{2}$ & & & 24.29 & & & 30.60 & & & 29.20 & \\
\hline $\mathrm{RC}_{3}$ & & & - & & & $95.5(122.6-4$ & 4686) & & - & \\
\hline $\mathrm{b} \pm$ & & & - & & & $1.9 \pm 0.5$ & & & - & \\
\hline 200 & & 7 & $0.96 \pm 0.11$ & $\mathrm{~N}$ & 360 & $0.78 \pm 0.49$ & $\mathrm{~N}$ & 21 & $0.89 \pm 0.32$ & $\mathrm{~N}$ \\
\hline 135 & & 6 & $0.97 \pm 0.13$ & $\mathrm{~N}$ & 60 & $0.97 \pm 0.13$ & $\mathrm{~N}$ & 16 & $0.92 \pm 0.33$ & $\mathrm{~N}$ \\
\hline 100 & & 4 & $0.98 \pm 0.13$ & $\mathrm{~N}$ & 30 & $0.98 \pm 0.09$ & $\mathrm{~N}$ & 10 & $0.95 \pm 0.20$ & $\mathrm{~N}$ \\
\hline 60 & 5 & 2 & $0.99 \pm 0.05$ & $\mathrm{~N}$ & 50 & $0.97 \pm 0.12$ & $\mathrm{~N}$ & 8 & $0.96 \pm 0.13$ & $\mathrm{~N}$ \\
\hline 40 & & 2 & $0.99 \pm 0.05$ & $\mathrm{~N}$ & 1 & $0.99 \pm 0.04$ & $\mathrm{~N}$ & 5 & $0.98 \pm 0.10$ & $\mathrm{~N}$ \\
\hline 10 & & 1 & $0.99 \pm 0.04$ & $\mathrm{~N}$ & 2 & $0.99 \pm 0.09$ & $\mathrm{~N}$ & 4 & $0.98 \pm 0.08$ & $\mathrm{~N}$ \\
\hline 3.5 & & 0 & $1.00 \pm 0.00$ & $\mathrm{~N}$ & 0 & $1.00 \pm 0.00$ & $\mathrm{~N}$ & 1 & $1.00 \pm 0.04$ & $\mathrm{~N}$ \\
\hline Con & trol & 0 & - & - & 0 & - & - & 1 & - & - \\
\hline $\operatorname{Pr}>$ & & & 0.0832 & & & 0.0217 & & & 0.0722 & \\
\hline $\mathrm{X}$ & & & 12.57 & & & 16.39 & & & 12.99 & \\
\hline $\mathrm{RC}$ & & & - & & & - & & & - & \\
\hline $\mathrm{b} \pm$ & & & - & & & - & & & - & \\
\hline 200 & & 15 & $0.93 \pm 0.10$ & $\mathrm{~N}$ & 20 & $0.89 \pm 0.32$ & $\mathrm{~N}$ & 16 & $0.91 \pm 0.31$ & $\mathrm{~N}$ \\
\hline 135 & & 4 & $0.99 \pm 0.11$ & $\mathrm{~N}$ & 60 & $0.97 \pm 0.16$ & $\mathrm{~N}$ & 13 & $0.93 \pm 0.37$ & $\mathrm{~N}$ \\
\hline 100 & & 1 & $1.01 \pm 0.04$ & $\mathrm{~N}$ & 20 & $0.99 \pm 0.05$ & $\mathrm{~N}$ & 9 & $0.95 \pm 0.20$ & $\mathrm{~N}$ \\
\hline 60 & 6 & 2 & $1.00 \pm 0.05$ & $\mathrm{~N}$ & 20 & $0.99 \pm 0.09$ & $\mathrm{~N}$ & 7 & $0.96 \pm 0.13$ & $\mathrm{~N}$ \\
\hline 40 & & 2 & $1.00 \pm 0.05$ & $\mathrm{~N}$ & 0 & $1.00 \pm 0.00$ & $\mathrm{~N}$ & 8 & $0.96 \pm 0.23$ & $\mathrm{~N}$ \\
\hline 10 & & 2 & $1.00 \pm 0.05$ & $\mathrm{~N}$ & 0 & $1.00 \pm 0.00$ & $\mathrm{~N}$ & 5 & $0.97 \pm 0.10$ & $\mathrm{~N}$ \\
\hline 3.5 & & 0 & $1.01 \pm 0.00$ & $\mathrm{~N}$ & 1 & $0.99 \pm 0.04$ & $\mathrm{~N}$ & 1 & $0.99 \pm 0.04$ & $\mathrm{~N}$ \\
\hline Con & trol & 2 & - & - & 0 & - & - & 0 & - & - \\
\hline $\operatorname{Pr}>$ & & & 0.0104 & & & 0.0133 & & & 0.3036 & \\
\hline $\mathrm{X}$ & & & 18.38 & & & 17.72 & & & 8.34 & \\
\hline $\mathrm{RC}$ & & & - & & & - & & & - & \\
\hline $\mathrm{b} \pm$ & & & - & & & - & & & - & \\
\hline 200 & & 17 & $0.91 \pm 0.35$ & $\mathrm{~N}$ & 330 & $0.81 \pm 0.27$ & $\mathrm{~N}$ & 19 & $0.90 \pm 0.47$ & $\mathrm{~N}$ \\
\hline 135 & & 12 & $0.94 \pm 0.15$ & $\mathrm{~N}$ & 10 & $0.96 \pm 0.24$ & $\mathrm{~N}$ & 9 & $0.96 \pm 0.16$ & $\mathrm{~N}$ \\
\hline 100 & & 11 & $0.94 \pm 0.23$ & $\mathrm{~N}$ & 1 & $1.01 \pm 0.04$ & $\mathrm{~N}$ & 6 & $0.97 \pm 0.22$ & $\mathrm{~N}$ \\
\hline 60 & 24 & 7 & $0.96 \pm 0.15$ & $\mathrm{~N}$ & 40 & $0.99 \pm 0.08$ & $\mathrm{~N}$ & 6 & $0.97 \pm 0.11$ & $\mathrm{~N}$ \\
\hline 40 & & 8 & $0.96 \pm 0.11$ & $\mathrm{~N}$ & 7 & $0.97 \pm 0.11$ & $\mathrm{~N}$ & 6 & $0.97 \pm 0.18$ & $\mathrm{~N}$ \\
\hline 10 & & 2 & $0.99 \pm 0.05$ & $\mathrm{~N}$ & 6 & $0.98 \pm 0.22$ & $\mathrm{~N}$ & 3 & $0.99 \pm 0.05$ & $\mathrm{~N}$ \\
\hline 3.5 & & 30 & $0.98 \pm 0.09$ & $\mathrm{~N}$ & 1 & $1.01 \pm 0.04$ & $\mathrm{~N}$ & 2 & $0.99 \pm 0.05$ & $\mathrm{~N}$ \\
\hline Con & trol & 0 & - & - & 2 & - & - & 1 & - & - \\
\hline $\operatorname{Pr}>$ & $X^{2}$ & & 0.0724 & & & 0.0087 & & & 0.4518 & \\
\hline $\mathrm{X}^{2}$ & & & 12.99 & & & 18.84 & & & 6.78 & \\
\hline $\mathrm{RC}$ & & & - & & & - & & & - & \\
\hline $\mathrm{b} \pm$ & & & - & & & - & & & - & \\
\hline
\end{tabular}

${ }^{1}$ Percentage repellency obtained from real data; ${ }^{2} \mathrm{IR}$ : repellency index; ${ }^{3}$ Clasification: R: Repellent; N: Neutral; ${ }^{4}$ Data not considered in probit analysis; ${ }^{5}$ Confidence intervals at $95 \%$, b: regression line slope, $\mathrm{s}$ : standard error.
$24 \mathrm{~h}$; consequently, it was not possible to estimate $\mathrm{RC}_{50}$ values.

The slope values of water and ethanolic extracts with repellent activity were superior to $1.0 \pm 0.2$, which indicate uniformity of population response to selection with the evaluated products. The low repellent activity of acetonic extracts in this study is attributed to their high volatility and low persistence (Schuster et al., 2009). This aspect coincides with other researchers who noted that non-polar extracts were less efficient than those with intermediate polarity; for this reason they emphasize the importance of improving formulation or microencapsulation of active ingredients to prevent their rapid degradation (Saito et al., 1989; Roel et al., 2000; Schuster et al., 2009). Based on the results obtained in this study and those reported by the previously mentioned researchers, there is a preference for intermediate polarity botanical extracts (Gómez et al., 1997; Cubillo et al., 1999). In contrast with the results with acetonic extracts, water and ethanolic extracts of common ragweed, dandelion, and wild radish, as well as the ethanolic extract of Mexican pepperleaf reduced the settling of greenhouse whiteflies on treated disks. Nevertheless, persistence and effectiveness of extracts varied as a function of concentration and time. This response already had been observed by Hoss and Gomero (1994), Iannacone (2008), and Morar et al. (2008) when they applied T. officinale extracts at rates of $100 \mathrm{mg} \mathrm{mL}^{-1}$ against various pests. The difference in the repellency response to ethanolic extracts compared with water extracts could be due to the concentration of active principles indicated by Ladd et al. (1978) and Leyva et al. (2009), who stated that the use of solvents with different polarities in the extraction and formulation modified the composition and concentration of active principles. The repellent activity and persistence of extracts therefore depend on the size and shape of the molecules in each product and, as these are joined and assembled in the sensorial receptors, they are present on the antennae of whiteflies (Wright, 1975). In our study, it was evident that the repellent activity of water extracts of $R$. raphanistrum and T. officinale and the ethanolic extract of $A$. artemisiifolia and $R$. raphanistrum gradually decreased over time. This is partly due to the rapid decomposition of the compounds caused by light, UV rays, temperature, rain, $\mathrm{pH}$, and microbial activity (Mulla and $\mathrm{Su}, 1999$ ); this aspect was also recorded by Cubillo et al. (1999) when evaluating neem products on Bemisia tabaci (Gennadius) and by Camarillo et al. (2009) in assay extracts of $T$. filifolia (Asteraceae) against T. vaporariorum. However, several authors state that repellent activity can recover over time due to temporal processes of desaturation and saturation of the chemoreceptors in the insect (Lenteren and Noldus, 1990; Camarillo et al., 2009).

\section{Mortality and oviposition inhibition in adults}

From all the evaluated extracts only the ethanolic extract 
Table 2. Mean repellency (\%) of adult whitefly Trialeurodes vaporariorum 3, 4, 5, 6, and $24 \mathrm{~h}$ after applying ethanolic extracts of four plant species.

\begin{tabular}{|c|c|c|c|c|c|c|c|c|c|c|c|c|c|}
\hline \multirow{2}{*}{$\begin{array}{l}\text { Conc. } \\
\left(\mathrm{mg} \mathrm{mL}^{-1}\right)\end{array}$} & \multirow[b]{2}{*}{ Hour } & \multicolumn{3}{|c|}{ Ambrosia artemisiifolia } & \multicolumn{3}{|c|}{ Piper auritum } & \multicolumn{3}{|c|}{ Raphanus raphanistrum } & \multicolumn{3}{|c|}{ Taraxacum officinale } \\
\hline & & Repel $(\%)^{1}$ & $\mathrm{RI}^{2}$ & $\mathrm{CL}^{3}$ & Repel. (\%) & RI & $\mathrm{CL}$ & Repel. (\%) & RI & CL & Repel. (\%) & RI & CL \\
\hline 200 & \multirow{8}{*}{3} & 62 & $0.59 \pm 0.24$ & $\mathrm{R}$ & 57 & $0.65 \pm 0.34$ & $\mathrm{R}$ & 76 & $0.42 \pm 0.15$ & $\mathrm{R}$ & 45.0 & $0.76 \pm 0.16$ & $\mathrm{R}$ \\
\hline 135 & & 47 & $0.74 \pm 0.36$ & $\mathrm{~N}$ & 47 & $0.74 \pm 0.19$ & $\mathrm{R}$ & 43 & $0.77 \pm 0.56$ & $\mathrm{~N}$ & 43.8 & $0.77 \pm 0.11$ & $\mathrm{R}$ \\
\hline 100 & & 42 & $0.78 \pm 0.21$ & $\mathrm{R}$ & 42 & $0.78 \pm 0.09$ & $\mathrm{R}$ & 35 & $0.84 \pm 0.41$ & $\mathrm{~N}$ & 38.8 & $0.82 \pm 0.43$ & $\mathrm{~N}$ \\
\hline 60 & & 37 & $0.82 \pm 0.11$ & $\mathrm{R}$ & 35 & $0.84 \pm 0.29$ & $\mathrm{~N}$ & 36 & $0.83 \pm 0.20$ & $\mathrm{~N}$ & 30.0 & $0.88 \pm 0.37$ & $\mathrm{~N}$ \\
\hline 40 & & 35 & $0.83 \pm 0.16$ & $\mathrm{R}$ & 22 & $0.93 \pm 0.28$ & $\mathrm{~N}$ & 27 & $0.89 \pm 0.30$ & $\mathrm{~N}$ & 31.3 & $0.87 \pm 0.37$ & $\mathrm{~N}$ \\
\hline 10 & & $25^{4}$ & $0.90 \pm 0.12$ & $\mathrm{~N}$ & $27^{4}$ & $0.89 \pm 0.25$ & $\mathrm{~N}$ & 21 & $0.93 \pm 0.08$ & $\mathrm{~N}$ & 18.8 & $0.95 \pm 0.25$ & $\mathrm{~N}$ \\
\hline 3.5 & & $26^{4}$ & $0.89 \pm 0.08$ & $\mathrm{R}$ & $21^{4}$ & $0.93 \pm 0.19$ & $\mathrm{~N}$ & 18 & $0.95 \pm 0.15$ & $\mathrm{~N}$ & 12.5 & $0.99 \pm 0.19$ & $\mathrm{~N}$ \\
\hline Control & & 9 & & & 10 & & & 10 & & & 11.2 & & \\
\hline $\operatorname{Pr}>X^{2}$ & & \multicolumn{3}{|c|}{$<0.0001$} & \multicolumn{3}{|c|}{0.0006} & \multicolumn{3}{|c|}{0.0013} & \multicolumn{3}{|c|}{0.0160} \\
\hline $\mathrm{X}^{2}$ & & & 29.89 & & & 25.59 & & & 13.59 & & & 17.22 & \\
\hline $\mathrm{RC}_{50}$ & & 187.5 & $.5(140.5-334$ & & 192. & $2.1(146.5-38$ & & & $4.3(58.9-1487$ & & & - & \\
\hline $\mathrm{b} \pm \mathrm{s}$ & & & $1.1 \pm 0.2$ & & & $1.2 \pm 0.3$ & & & $1.0 \pm 0.2$ & & & - & \\
\hline 200 & & 43 & $0.75 \pm 0.34$ & $\mathrm{~N}$ & 38 & $0.80 \pm 0.24$ & $\mathrm{~N}$ & 51 & $0.69 \pm 0.28$ & $\mathrm{R}$ & 27.5 & $0.86 \pm 0.06$ & $\mathrm{R}$ \\
\hline 135 & & 31 & $0.85 \pm 0.22$ & $\mathrm{~N}$ & 25 & $0.90 \pm 0.34$ & $\mathrm{~N}$ & 24 & $0.89 \pm 0.43$ & $\mathrm{~N}$ & 25.5 & $0.88 \pm 0.39$ & $\mathrm{~N}$ \\
\hline 100 & & 32 & $0.84 \pm 0.15$ & $\mathrm{R}$ & 15 & $0.96 \pm 0.17$ & $\mathrm{~N}$ & 17 & $0.94 \pm 0.29$ & $\mathrm{~N}$ & 12.5 & $0.96 \pm 0.11$ & $\mathrm{~N}$ \\
\hline 60 & 4 & 22 & $0.91 \pm 0.21$ & $\mathrm{~N}$ & 13 & $0.97 \pm 0.30$ & $\mathrm{~N}$ & 20 & $0.92 \pm 0.12$ & $\mathrm{~N}$ & 17.5 & $0.93 \pm 0.19$ & $\mathrm{~N}$ \\
\hline 40 & & 19 & $0.93 \pm 0.30$ & $\mathrm{~N}$ & 17 & $0.95 \pm 0.23$ & $\mathrm{~N}$ & 13 & $0.97 \pm 0.24$ & $\mathrm{~N}$ & 10.0 & $0.97 \pm 0.24$ & $\mathrm{~N}$ \\
\hline 10 & & 19 & $0.93 \pm 0.22$ & $\mathrm{~N}$ & 14 & $0.97 \pm 0.13$ & $\mathrm{~N}$ & 14 & $0.96 \pm 0.16$ & $\mathrm{~N}$ & 11.3 & $0.96 \pm 0.29$ & $\mathrm{~N}$ \\
\hline 3.5 & & 12 & $0.97 \pm 0.11$ & $\mathrm{~N}$ & 12 & $0.98 \pm 0.13$ & $\mathrm{~N}$ & 7 & $1.00 \pm 0.13$ & $\mathrm{~N}$ & 10.0 & $0.97 \pm 0.16$ & $\mathrm{~N}$ \\
\hline Control & & 6 & & & 8 & & & 7 & & & 5.0 & & \\
\hline $\operatorname{Pr}>X^{2}$ & & & 0.0039 & & & 0.0457 & & & 0.0098 & & & 0.0712 & \\
\hline $\mathrm{X}^{2}$ & & & 20.89 & & & 14.33 & & & 18.52 & & & 13.04 & \\
\hline $\mathrm{RC}_{50}$ & & & - & & & - & & & - & & & - & \\
\hline $\mathrm{b} \pm \mathrm{s}$ & & & - & & & - & & & - & & & - & \\
\hline 200 & & 31 & $0.84 \pm 0.32$ & $\mathrm{~N}$ & 15 & $0.93 \pm 0.28$ & $\mathrm{~N}$ & 31 & $0.84 \pm 0.34$ & $\mathrm{~N}$ & 16.3 & $0.92 \pm 0.11$ & $\mathrm{~N}$ \\
\hline 135 & & 17 & $0.93 \pm 0.17$ & $\mathrm{~N}$ & 11 & $0.96 \pm 0.16$ & $\mathrm{~N}$ & 13 & $0.95 \pm 0.37$ & $\mathrm{~N}$ & 13.8 & $0.93 \pm 0.25$ & $\mathrm{~N}$ \\
\hline 100 & & 23 & $0.86 \pm 0.24$ & $\mathrm{~N}$ & 9 & $0.97 \pm 0.20$ & $\mathrm{~N}$ & 13 & $0.95 \pm 0.36$ & $\mathrm{~N}$ & 3.8 & $0.99 \pm 0.11$ & $\mathrm{~N}$ \\
\hline 60 & 5 & 18 & $0.93 \pm 0.18$ & $\mathrm{~N}$ & 5 & $0.99 \pm 0.14$ & $\mathrm{~N}$ & 13 & $0.95 \pm 0.32$ & $\mathrm{~N}$ & 5.0 & $0.98 \pm 0.16$ & $\mathrm{~N}$ \\
\hline 40 & & 17 & $0.93 \pm 0.27$ & $\mathrm{~N}$ & 2 & $1.01 \pm 0.09$ & $\mathrm{~N}$ & 8 & $0.98 \pm 0.11$ & $\mathrm{~N}$ & 3.8 & $0.99 \pm 0.17$ & $\mathrm{~N}$ \\
\hline 10 & & 18 & $0.93 \pm 0.25$ & $\mathrm{~N}$ & 4 & $0.99 \pm 0.09$ & $\mathrm{~N}$ & 5 & $0.99 \pm 0.10$ & $\mathrm{~N}$ & 5.0 & $0.98 \pm 0.13$ & $\mathrm{~N}$ \\
\hline 3.5 & & 10 & $0.97 \pm 0.21$ & $\mathrm{~N}$ & 3 & $1.00 \pm 0.09$ & $\mathrm{~N}$ & 2 & $0.98 \pm 0.05$ & $\mathrm{~N}$ & 2.5 & $0.99 \pm 0.11$ & $\mathrm{~N}$ \\
\hline Control & & 5 & & & 3 & & & 4 & & & 1.3 & & \\
\hline $\operatorname{Pr}>X^{2}$ & & & 0.0749 & & & 0.3817 & & & 0.0689 & & & 0.0926 & \\
\hline $\mathrm{X}^{2}$ & & & 12.89 & & & 7.47 & & & 13.13 & & & 12.25 & \\
\hline $\mathrm{RC}_{50}$ & & & - & & & - & & & - & & & - & \\
\hline $\mathrm{b} \pm \mathrm{s}$ & & & - & & & - & & & - & & & - & \\
\hline 200 & & 18 & $0.91 \pm 0.45$ & $\mathrm{~N}$ & 11 & $0.95 \pm 0.22$ & $\mathrm{~N}$ & 15 & $0.93 \pm 0.17$ & $\mathrm{~N}$ & 10.0 & $0.95 \pm 0.24$ & $\mathrm{~N}$ \\
\hline 135 & & 9 & $0.96 \pm 0.27$ & $\mathrm{~N}$ & 2 & $0.99 \pm 0.09$ & $\mathrm{~N}$ & 3 & $1.00 \pm 0.09$ & $\mathrm{~N}$ & 10.0 & $0.95 \pm 0.16$ & $\mathrm{~N}$ \\
\hline 100 & & 6 & $0.97 \pm 0.13$ & $\mathrm{~N}$ & 0 & $1.01 \pm 0.00$ & $\mathrm{~N}$ & 2 & $1.01 \pm 0.05$ & $\mathrm{~N}$ & 5.0 & $0.97 \pm 0.16$ & $\mathrm{~N}$ \\
\hline 60 & 6 & 3 & $0.99 \pm 0.09$ & $\mathrm{~N}$ & 2 & $0.99 \pm 0.05$ & $\mathrm{~N}$ & 3 & $1.00 \pm 0.05$ & $\mathrm{~N}$ & 6.3 & $0.96 \pm 0.17$ & $\mathrm{~N}$ \\
\hline 40 & & 4 & $0.98 \pm 0.12$ & $\mathrm{~N}$ & 1 & $1.00 \pm 0.04$ & $\mathrm{~N}$ & 4 & $0.99 \pm 0.08$ & $\mathrm{~N}$ & 5.0 & $0.97 \pm 0.09$ & $\mathrm{~N}$ \\
\hline 10 & & 3 & $0.99 \pm 0.09$ & $\mathrm{~N}$ & 3 & $0.99 \pm 0.09$ & $\mathrm{~N}$ & 2 & $1.01 \pm 0.05$ & $\mathrm{~N}$ & 3.8 & $0.98 \pm 0.06$ & $\mathrm{~N}$ \\
\hline 3.5 & & 1 & $1.00 \pm 0.04$ & $\mathrm{~N}$ & 0 & $1.01 \pm 0.00$ & $\mathrm{~N}$ & 3 & $1.00 \pm 0.05$ & $\mathrm{~N}$ & 3.8 & $0.98 \pm 0.11$ & $\mathrm{~N}$ \\
\hline Control & & 1 & & & 1 & & & 3 & & & 0.0 & & \\
\hline $\operatorname{Pr}>X^{2}$ & & & 0.6350 & & & 0.2335 & & & 0.3028 & & & 0.3882 & \\
\hline $\mathrm{X}^{2}$ & & & 5.20 & & & 9.27 & & & 8.35 & & & 7.40 & \\
\hline $\mathrm{RC}_{50}$ & & & - & & & - & & & - & & & - & \\
\hline $\mathrm{b} \pm \mathrm{s}$ & & & - & & & - & & & - & & & - & \\
\hline 200 & & 18 & $0.92 \pm 0.26$ & $\mathrm{~N}$ & 3 & $0.98 \pm 0.09$ & $\mathrm{~N}$ & 16 & $0.95 \pm 0.36$ & $\mathrm{~N}$ & 15.0 & $0.93 \pm 0.16$ & $\mathrm{~N}$ \\
\hline 135 & & 16 & $0.93 \pm 0.28$ & $\mathrm{~N}$ & 2 & $0.99 \pm 0.09$ & $\mathrm{~N}$ & 14 & $0.97 \pm 0.57$ & $\mathrm{~N}$ & 18.8 & $0.91 \pm 0.28$ & $\mathrm{~N}$ \\
\hline 100 & & 19 & $0.91 \pm 0.48$ & $\mathrm{~N}$ & 2 & $0.99 \pm 0.05$ & $\mathrm{~N}$ & 5 & $1.02 \pm 0.12$ & $\mathrm{~N}$ & 7.5 & $0.97 \pm 0.06$ & $\mathrm{~N}$ \\
\hline 60 & 24 & 17 & $0.92 \pm 0.54$ & $\mathrm{~N}$ & 0 & $1.00 \pm 0.00$ & $\mathrm{~N}$ & 8 & $1.00 \pm 0.26$ & $\mathrm{~N}$ & 5.0 & $0.99 \pm 0.09$ & $\mathrm{~N}$ \\
\hline 40 & & 18 & $0.92 \pm 0.40$ & $\mathrm{~N}$ & 0 & $1.00 \pm 0.00$ & $\mathrm{~N}$ & 7 & $1.01 \pm 0.11$ & $\mathrm{~N}$ & 10.0 & $0.96 \pm 0.16$ & $\mathrm{~N}$ \\
\hline 10 & & 10 & $0.96 \pm 0.39$ & $\mathrm{~N}$ & 0 & $1.00 \pm 0.00$ & $\mathrm{~N}$ & 9 & $0.99 \pm 0.22$ & $\mathrm{~N}$ & 7.5 & $0.97 \pm 0.14$ & $\mathrm{~N}$ \\
\hline 3.5 & & 11 & $0.96 \pm 0.39$ & $\mathrm{~N}$ & 1 & $0.99 \pm 0.04$ & $\mathrm{~N}$ & 2 & $1.03 \pm 0.09$ & $\mathrm{~N}$ & 8.8 & $0.97 \pm 0.14$ & $\mathrm{~N}$ \\
\hline Control & & 3 & & & 0 & & & 8 & & & 2.5 & & \\
\hline $\operatorname{Pr}>X^{2}$ & & & 0.7007 & & & 0.3112 & & & 0.7007 & & & 0.1789 & \\
\hline $\mathrm{X}^{2}$ & & & 4.67 & & & 8.25 & & & 4.67 & & & 10.18 & \\
\hline $\mathrm{RC}_{50}$ & & & - & & & - & & & - & & & - & \\
\hline $\mathrm{b} \pm \mathrm{s}$ & & & - & & & - & & & - & & & - & \\
\hline
\end{tabular}

${ }^{1}$ Percentage repellency obtained from real data; ${ }^{2}$ IR: repellency index; ${ }^{3}$ Clasification: R: Repellent; N: Neutral; ${ }^{4}$ Data not considered in probit analysis; ${ }^{5}$ Confidence intervals at $95 \%$, b: regression line slope, s: standard error.

of $R$. raphanistrum and water and ethanolic extracts of $P$. auritum were toxic against the greenhouse whitefly although none of the assessed extracts caused absolute mortality. Toxicity had a positive relationship with concentration. The highest mortality was recorded by applying ethanolic extracts of $P$. auritum $(66 \%)$ and $R$. raphanistrum $(56 \%)\left(\mathrm{P} \leq 0.05 ; \mathrm{X}^{2}=25.15\right.$ and 29.65 , respectively) at $200 \mathrm{mg} \mathrm{mL}^{-1}$; however, both extracts also showed a significant effect $(\geq 30 \%)$ at $100 \mathrm{mg} \mathrm{mL}^{-1}$. An $\mathrm{LC}_{50}$ of $116.0 \mathrm{mg} \mathrm{mL}^{-1}$ was estimated with the ethanolic 
extract of $P$. auritum followed by $R$. raphanistrum (185.2 $\mathrm{mg} \mathrm{mL} \mathrm{m}^{-1}$ ) (Table 3).

The slopes recorded for the ethanolic extracts of $P$. auritum and $R$. raphanistrum were $1.7 \pm 0.2$ and $1.4 \pm 0.3$, respectively; these values indicate that the greenhouse whitefly population responded uniformly to selection with any one of the applied extracts.

In general, $T$. vaporariorum adult mortality caused by the extracts was mainly associated with the dissolvent used for extraction and the concentration tested. It might be explained by the difference in composition of active principles present in different plant structures since some are non-polar substances that are difficult to extract in water (Delgado and Cuca, 2007; Scott et al., 2008; Camarillo et al., 2009). The toxicity of extracts is also due to a differential concentration of active principles in plant structures, a property that was demonstrated by Weber et al. (1994) in Zabrotes subfasciatus (Boheman) and Camarillo et al. (2009) in T. vaporariorum, who showed that flower oils of Tagetes minuta and T. filifolia, respectively, were more active than extracts from other parts of the plant because flowers have a greater number of terpenes of low molecular weight, such as trans-anethole or alilanisol. This finding was also noted by Sáez et al. (1998) with leaf extract of $P$. auritum against the fruit fly (Drosophila melanogaster, Meig.), which is related to a higher safrol concentration (Sánchez et al., 2009).

Greenhouse whitefly oviposition was also adversely affected by water extracts of $P$. auritum and $R$. raphanistrum, ethanolic extracts of $A$. artemisiifolia, $P$. auritum and $R$. raphanistrum, and acetonic extract of $P$. auritum $(\mathrm{P} \leq 0.05)$. Water extracts of $R$. raphanistrum and $P$. auritum, applied at $200 \mathrm{mg} \mathrm{mL}^{-1}$, were the most effective to inhibit oviposition in $76.15 \%$ and $72.04 \%$, respectively (Table 4).

Acetonic and ethanolic extracts of P. auritum at $100 \mathrm{mg}$ $\mathrm{mL}^{-1}$ also interrupted oviposition by approximately $50 \%$; however, the acetonic extract caused phytotoxicity at that concentration and this limits its application in greenhouse

Table 3. Mortality mean (\%) of adult whitefly Trialeurodes vaporariorum $24 \mathrm{~h}$ after applying water and ethanolic extracts of Piper auritum and Raphanus raphanistrum.

\begin{tabular}{lcccc}
\hline \multirow{2}{*}{$\begin{array}{c}\text { Concentration } \\
\left(\mathrm{mg} \mathrm{mL}^{-1}\right)\end{array}$} & Water & & \multicolumn{2}{c}{ Ethanolic } \\
\cline { 2 - 3 } \cline { 5 - 5 } & P. auritum & & P. auritum & R. raphanistrum \\
\hline 200 & $35.0^{1}$ & & 66.0 & 56.0 \\
140 & 29.0 & & 55.0 & 40.0 \\
100 & 24.0 & & 49.0 & 36.0 \\
60 & 16.0 & 25.0 & 27.0 \\
40 & 16.0 & & 24.0 & $11.0^{2}$ \\
10 & 7.0 & & - & 12.0 \\
Control & 2.0 & & 3.0 & 2.0 \\
\hline Pr $>\mathrm{X}^{2}$ & 0.0002 & & 0.0001 & $<0.0001$ \\
$\mathrm{X}^{2}$ & 26.29 & 25.15 & 29.65 \\
\hline $\mathrm{LC}_{50}\left(\mathrm{mg} \mathrm{mL}^{-1}\right)$ & - & 116.0 & 185.2 \\
\multicolumn{1}{c}{$\mathrm{b} \pm \mathrm{s}$} & - & $(99.7-139.2)^{3}$ & $(147.2-300.2)$ \\
\hline
\end{tabular}

- Concentration not evaluated, ${ }^{1}$ Percentage of mortality taken from real data, ${ }^{2}$ Data not considered in probit analysis, ${ }^{3}$ Confidence intervals at 95\%, b: regression line slope, s: standard error.
Table 4. Oviposition inhibition mean $(\%)$ in adult whitefly Trialeurodes vaporariorum $24 \mathrm{~h}$ after applying water and ethanolic and acetonic plant extracts.

\begin{tabular}{|c|c|c|c|c|c|c|}
\hline \multirow[b]{2}{*}{$\begin{array}{l}\text { Concentration } \\
\left(\mathrm{mg} \mathrm{mL}^{-1}\right)\end{array}$} & \multicolumn{6}{|c|}{ Water } \\
\hline & $\begin{array}{c}\text { Ambrosia } \\
\text { artemisiifolia }\end{array}$ & \multicolumn{2}{|c|}{$\begin{array}{c}\text { Piper } \\
\text { auritum }\end{array}$} & \multicolumn{2}{|c|}{$\begin{array}{c}\text { Raphanus } \\
\text { raphanistrum }\end{array}$} & $\begin{array}{l}\text { Taraxacum } \\
\text { officinale }\end{array}$ \\
\hline 200 & 38.84 & \multicolumn{2}{|c|}{72.04} & \multicolumn{2}{|c|}{76.15} & 42.98 \\
\hline 135 & 28.93 & \multicolumn{2}{|c|}{43.01} & \multicolumn{2}{|c|}{57.69} & 42.11 \\
\hline 100 & 26.45 & \multicolumn{2}{|c|}{36.55} & \multicolumn{2}{|c|}{50.77} & 11.40 \\
\hline 60 & 14.05 & \multicolumn{2}{|c|}{$15.05^{1}$} & \multicolumn{2}{|c|}{53.85} & 12.28 \\
\hline 40 & 7.44 & \multicolumn{2}{|c|}{37.63} & \multicolumn{2}{|c|}{23.08} & $-0.88^{1}$ \\
\hline 10 & 0.83 & \multicolumn{2}{|c|}{11.83} & \multicolumn{2}{|c|}{$5.38^{1}$} & $-6.14^{1}$ \\
\hline 3.5 & $-10.74^{1}$ & \multicolumn{2}{|c|}{-} & \multicolumn{2}{|c|}{10.00} & 2.63 \\
\hline $\operatorname{Pr}>X^{2}$ & 0.4786 & \multicolumn{2}{|c|}{0.0201} & \multicolumn{2}{|c|}{0.0002} & 0.0234 \\
\hline $\mathrm{X}^{2}$ & 6.5374 & \multicolumn{2}{|c|}{15.0243} & \multicolumn{2}{|c|}{27.7482} & 16.1940 \\
\hline $\begin{array}{l}\mathrm{IOC}_{50} \\
\mathrm{~b} \pm \mathrm{s}\end{array}$ & $\begin{array}{l}- \\
-\end{array}$ & $\begin{array}{r}119 \\
(36.8-1 \\
1.0 \pm\end{array}$ & $\begin{array}{l}9.0 \\
191.1)^{2} \\
0.3\end{array}$ & $\begin{array}{r}7 \\
(35.2 \\
1.1\end{array}$ & $\begin{array}{l}77.3 \\
.2-198.2) \\
1 \pm 0.2\end{array}$ & $\begin{array}{l}- \\
-\end{array}$ \\
\hline $\mathrm{b} \pm \mathrm{s}$ & \multicolumn{5}{|c|}{ Ethanolic } & Acetonic \\
\hline $\begin{array}{l}\text { Concentration } \\
\left(\mathrm{mg} \mathrm{mL}^{-1}\right)\end{array}$ & $\begin{array}{c}\text { Ambrosia } \\
\text { artemisiifolia }\end{array}$ & $\begin{array}{c}\text { Piper } \\
\text { auritum }\end{array}$ & $\begin{array}{r}\text { Raph } \\
\text { raphan }\end{array}$ & $\begin{array}{l}\text { anus } \\
\text { istrum }\end{array}$ & $\begin{array}{l}\text { Taraxacum } \\
\text { officinale }\end{array}$ & $\begin{array}{c}\text { Piper } \\
\text { auritum }\end{array}$ \\
\hline 200 & 61.62 & 69.51 & 64 & & 60.87 & 68.18 \\
\hline 135 & 41.41 & 50.00 & 36 & & 39.13 & $48.86^{1}$ \\
\hline 100 & 45.45 & 34.15 & 28 & & 50.72 & 53.41 \\
\hline 60 & 31.31 & $3.66^{1}$ & 13 & & 44.93 & 40.91 \\
\hline 40 & 31.31 & $10.98^{1}$ & -15 & & 30.43 & 47.72 \\
\hline 10 & 14.14 & 15.85 & & $68^{1}$ & 33.33 & 6.82 \\
\hline 3.5 & $-17.17^{1}$ & 17.07 & -11 & & 7.25 & $-12.50^{1}$ \\
\hline $\operatorname{Pr}>X^{2}$ & 0.0039 & 0.0169 & 0.0 & 37 & 0.1181 & 0.0011 \\
\hline $\mathrm{X}^{2}$ & 20.9305 & 17.0757 & 17.6 & 493 & 11.5042 & 22.2622 \\
\hline & 146.6 & 123.3 & 15 & & 94.8 & 89.1 \\
\hline $\mathrm{IOC}_{50}$ & $(110.8-219.5)$ & (26.4-2584) & $(141.6$ & 186.8) & $(43.1-579.1)$ & (47.7-225.6) \\
\hline $\mathrm{b} \pm \mathrm{s}$ & $0.9 \pm 0.1$ & $0.7 \pm 0.2$ & $2.7=$ & 0.3 & $0.7 \pm 0.1$ & $1.2 \pm 0.2$ \\
\hline
\end{tabular}

- Concentration evaluated, ${ }^{1}$ Data not considered in the probit analysis, ${ }^{2}$ Confidence intervals at $95 \%$, b: linear regression slope, s: standard error; $\mathrm{IOC}_{50}$ : inhibition of oviposition by $50 \%$.

whitefly management. For this reason, it is convenient to use the water extract of $R$. raphanistrum, which, in addition, is not phytotoxic and reduces oviposition at concentrations of $60 \mathrm{mg} \mathrm{mL}^{-1}$. Surprisingly, water and ethanolic extracts of A. artemisiifolia and acetonic extract of $P$. auritum at $3.5 \mathrm{mg} \mathrm{mL}^{-1}$ and water extract of $T$. officinale and ethanolic extract of $R$. raphanistrum at 3.5 to $4.0 \mathrm{mg} \mathrm{mL}^{-1}$ stimulated oviposition; this is probably in response to the stress caused in the female by sublethal concentrations and treating this privilege survival through a higher oviposition rate (Abdullah et al., 2006) (Table 4).

From all the evaluated extracts, the water extract of $R$. raphanistrum was the most effective to inhibit egglaying $\left(\mathrm{IOC}_{50}=77.3 \mathrm{mg} \mathrm{mL}^{-1}\right)$; this was followed by the ethanolic extract of T. officinale $\left(94.8 \mathrm{mg} \mathrm{mL}^{-1}\right)$ and the acetonic extract of P. auritum ( $89.1 \mathrm{mg} \mathrm{mL}^{-1}$ ) (Table 4).

All solvent extract plant substances and the more polar solvents extract hydrophilic substances, while the less polar solvents extract hydrophobic molecules; for this reason several authors have found better results with extracts prepared with non-polar or intermediate polarity (ethanolic) solvents (Ascher et al., 1984; Saito et al., 1989; Roel et al., 2000) as observed in the present study. In spite of this, the use of non-polar solvents is limited to field application because of its high cost, persistence, low availability, as well as the risk implied by its flammability (Rodríguez and Vendramim, 2008). 
Even though a linear concentration-inhibition response was obtained with some water, ethanolic, and acetonic extracts, it was also evident that low concentrations stimulated oviposition. This behavior was observed by Gómez et al. (1997), who reported a $13 \%$ increase in oviposition of B. tabaci after applying the commercial product Neem Oil at $0.03 \mathrm{mg} \mathrm{mL}^{-1}$, and Camarillo et al. (2009) when exposing T. vaporariorum adults to trans-anethole and T. filifolia floral oil concentrations lower than 3.5 and $0.1 \mathrm{mg} \mathrm{mL}^{-1}$, respectively. The reduction in oviposition rates in some insects is due to the disturbance in the activity of chemoreceptors or the integration of information during the process of search and host acceptance generated by pesticides and extracts (Umoru et al., 1996; Desneux et al., 2007). On the other hand, higher rates of oviposition are also associated with the fly's preference for plants with high contents of $\mathrm{N}$, sugars, amino acids in the phloem, and low $\mathrm{pH}$ as was demonstrated in B. tabaci in cotton plants treated with different rates of fenvalerate and acephate (Abdullah et al., 2006).

\section{CONCLUSIONS}

The results of this study reveal the effectiveness of water and ethanolic extracts from different plant species to manage whitefly populations. The effects had a positive relationship with concentration. Water and ethanolic extracts of Raphanus raphanistrum and Piper auritum at $200 \mathrm{mg} \mathrm{mL}^{-1}$ are promising for Trialeurodes vaporariorum management since these extracts caused an immediate toxic effect; at the same time, they showed the best repellent activity and significantly inhibited oviposition. However, it is essential to define and apply effective concentrations since lower concentrations of 60 $\mathrm{mg} \mathrm{mL}^{-1}$ caused oviposition stimulation. For this reason, it is important to continue the search for the best method to extract and identify active principles, to define the mode of action, and to know the influence of environmental conditions, as well as compatibility with natural enemies and control alternatives to clarify their real practical use.

\section{LITERATURE CITED}

Abbott, W.S. 1925. A method of computing the effectiveness of an insecticide. Journal of Economic Entomology 18:165-267.

Abdullah, N.M.M., J. Singh, and B. Sohal. 2006. Behavioral hormoligosis in oviposition preference of Bemisia tabaci on cotton. Pesticide Biochemistry Physiology 84:10-16.

Agnihotri, N.P. 1999. Pesticide safety evaluation and monitoring. p. 9-23. Division of Agriculture Chemicals, Indian Agricultural Research Institute New Delhi, India.

Altieri, M.A. 1995. Biodiversity and biocontrol: Lessons from insect pest management. Advances in Plant Pathology 11:191-206.

Ascher, K.R., M. Eliyahy, N.E. Nemny, and J. Meisner. 1984. Neem seed kernel extract as an inhibitor or growth and fecundity in Spodoptera littoralis. p. 331-344. In Schmutterer, H., and R.S. Ascher (eds.) Natural pesticides from the neem tree (Azadirachta indica A. Juss) and other tropical plants. Proceeding Second
International Neem Conference, Rauischholzhausen. 25-28 May 1983. German Agency for Technical Cooperation, Eschborn, Germany.

Calderón, G., y J. Rzedowski. 2001. Flora fanerogámica del Valle de México. 1406 p. $2^{\mathrm{a}}$ ed. Instituto de Ecología, A.C. y Comisión Nacional para el Conocimiento y Uso de la Biodiversidad, Pátzcuaro, Michoacán, México.

Camarillo, R.G., L.D. Ortega, M.A. Serrato, and C. Rodríguez. 2009. Actividad biológica de Tagetes filifolia (Asteraceae) en Trialeurodes vaporariorum (Hemiptera: Aleyrodidae). Revista Colombiana de Entomología 35:177-184.

Cubillo, D., G. Sanabria, and L. Hilje. 1999. Evaluación de repelencia y mortalidad causada por insecticidas comerciales y extractos vegetales sobre Bemisia tabaci. Manejo Integrado de Plagas 53:65-72.

Delgado, A.W., and S.L.E. Cuca. 2007. Composición química del aceite esencial de Piper hispidum. Revista de Productos Naturales $1: 5-8$

Desneux, N., A. Decourtye, and M.J. Delpuech. 2007. The sublethal effects of pesticides on beneficial arthropods. Annual Review of Entomology 52:81-106.

García, R.A., A.M. Leyva, R.J.M. Martínez, and E.E. Stashenko. 2007. Determinación de la composición química y actividad antioxidante in vitro del aceite esencial de Piper auritum Kunth (Piperaceae) difundida en la costa colombiana. Scientia et Technica 13(33):439-442.

Gómez, P., G.D. Cubillo, A. Mora, and L. Hilje. 1997. Evaluación de posibles repelentes de Bemisia tabaci: II. Extractos vegetales. Manejo Integrado de Plagas (Costa Rica) 46:17-25.

Grainge, M., and S. Ahmed. 1988. Handbook of plants with pestcontrol properties. 470 p. John Wiley and Sons, New York, USA.

Hoss, R., y L. Gomero. 1994. Efecto de extractos vegetales sobre la regulación de plagas del maíz, con énfasis en "cardo santo" (Argemone mexicana). p. 119-133. In Gomero, O.L. (ed.) Plantas para proteger cultivos. Tecnología para controlar plagas y enfermedades. Red de Acción en Alternativas al uso de Agroquímicos, Lima, Perú.

Iannacone, O.J. 2008. Actividad insecticida y repelente de plantas en el gorgojo del maíz Sitophilus zeamais. Scientia 10(10):171-179.

Jbilou, R., A. Ennabili, and F. Sayah. 2006. Insecticidal activity of four medicinal plant extracts against Tribolium castaneum (Herbst) (Coleoptera: Tenebrionidae). African Journal of Biotechnology 5:936-940.

Jones, R.D. 2003. Plant viruses transmitted by whiteflies. European Journal of Plant Pathology 109:195-219.

Jovanović, Z., M. Kostić, and Z. Popović. 2007. Grainprotective properties of herbal extracts against the bean weevil Acanthoscelides obtectus Say. Industrial Crops and Products 26:100-104.

Ladd, T.L., M. Jacobson, and C.R. Buriff. 1978. Japanese beetles; extracts from neem seeds as feeding deterrents. Journal of Economic Entomology 71:810-813.

Lenteren, V.J.C., and J.P.L. Noldus. 1990. Whitefly-plant relationships: behavioral an ecological aspects. p. 47-80. In Gerling, D. (ed.) Whiteflies: Their bionomics, pest status and management. Intercept, Andover, UK.

Leyva, M., C.M. Marquetti, J.E. Tacoronte, R. Scull, O. Tiomno, A. Mesa, et al. 2009. Actividad larvicida de aceites esenciales de plantas contra Aedes aegypti (L.) (Diptera: Culicidae). Revista Biomédica 20:5-13.

Lin, H., M. Kogan, and D. Fischer. 1990. Induced resistance in soybean to the Mexican bean beetle (Coleoptera: Coccinellidae): comparisons of inducing factors. Environmental Entomology 19:1852-1857.

Martin, J.H. 1987. An identification guide to common whitefly pest species of the World (Homoptera: Aleyrodidae). Tropical Pest Management 33:298-322.

Martin, J.H., and L.A. Mound. 2007. An annotated check list of the world's whiteflies (Insecta: Hemiptera: Aleyrodidae). Zootaxa 1492:1-84 
McCutcheon, G.S., M.A. Simmons, and K.L. Norsworthy. 2009 Effect of wild radish on preimaginal development of Diabrotica balteata and Agrotis ipsilon. Journal of Sustainable Agriculture 33:119-127.

Morar, G., C. Sîrbu, and I. Oltean. 2008. Effect of the hydroalcoholic extracts from plants on Colorado beetle (Leptinotarsa decemlineata, Say). Note II. ProEnvironment 2:46-49.

Mulla, M.S., and T. Su. 1999. Activity of biological effects of neem products against arthropods of medical and veterinary importance. Journal of the American Mosquito Control Association 15:133152 .

Ortega, L.D. 2008. Resistencia de moscas blancas a insecticidas. p. 57-67. In S. Infante G. (ed.) Moscas blancas temas selectos sobre su manejo. Colegio de Postgraduados. Mundi Prensa, México, D.F.

Ortega Arenas, L.D., A.L. Tejeda, J.C.R. Maciel, C.R. Hernández, R.A. Rosas, y N.M. Bárcenas. 1998. Susceptibilidad a insecticidas en adultos de mosquita blanca Trialeurodes vaporariorum (West.) (Homoptera: Aleyrodidae) de Tepoztlán, Morelos, México. Agrociencia 32:249-254.

Parmar, V., S. Subhash, C. Jain, K.S. Bisht, R. Jain, P. Taneja, et al. 1997. Phytochemistry of the genus Piper. Phytochemistry 46:597673.

Rodríguez, H.C. 1998. Recetas de plantas contra mosca blanca. p. 49-67. In C. Rodríguez H. (ed.) Memorias del I Simposio Internacional y IV Nacional sobre Sustancias Vegetales y Minerales en el Combate de Plagas. Colegio de PostgraduadosBenemérita Universidad Autónoma de Puebla, Acapulco, Guerrero, México.

Rodríguez, H.C. 2000. Plantas contra plagas: potencial práctico del ajo, anona, nim, chile y tabaco. 133 p. Red de Acción sobre Plaguicidas y Alternativas en México (RAPAM) y Universidad Autónoma Metropolitana-Unidad Xochimilco, México, DF.

Rodríguez, H.C., y J.D. Vendramim 2008. Substancias vegetales para el manejo de las moscas blancas, p. 83-102. In S. Infante G. (ed.) Moscas blancas temas selectos sobre su manejo. Colegio de Postgraduados, Mundi Prensa, México, D.F.
Roel, A.R., J.D. Vendramim, S.T.R. Frighetto, e N. Frighetto 2000. Atividade tóxica de extratos orgânicos de Trichilia pallida (Swartz) (Meliaceae) sobre Spodoptera frugiperda (J.E. Smith). Anais da Sociedade Entomológica do Brasil 29:799-808.

Sáez, J., H. Granados, E.M. Moreno, and A.C. Pelaez. 1998. In vitro array type standard for the evaluation of insecticide activity in Piper auritum leaves (Piperaceae). Afinidad 55:363-368.

Saito, M.L., F. Oliveira, D. Fell, P.A. Takematsu, T. Jocys, e J.L. Oliveira. 1989. Verificação da atividade inseticida de alguns vegetais brasileiros. Arquivos do Instituto Biológico São Paulo 56(1/2):53-59.

Sánchez, Y., O. Pino, M.T. Correa, E. Naranjo, y A. Iglesia. 2009 Estudio químico y microbiológico del aceite esencial de Piper auritum Kunth (Caisimón de anís). Revista de Protección Vegetal 24(1):39-46.

SAS Institute. 1999. The SAS System for Windows v.8. SAS Institute, Cary, North Carolina, USA.

Schuster, D.J., S. Thompson, L.D. Ortega A., and J.E. Polston 2009. Laboratory evaluation of products to reduce settling of sweet potato whitefly adults. Journal of Economic Entomology 102:1482-1489.

Scott, I.A., H.R. Jensen, B.J.R. Philogene, and J.T. Arnason. 2008 A review of Piper spp. (Piperaceae) phytochemistry, insecticidal activity and mode of action. Phytochemistry Reviews 7:65-75.

Umoru, P.A., W. Powell, and S.J. Clark. 1996. Effect of pirimicarb on the foraging behaviour of Diaeretiella rapae (Hym. Brac) on hostfree and infested oilseed rape plants. Bulletin of Entomological Research 86:193-210.

Weber, D.K., C.D. Wells, F.V. Dunkel, W. Bertsch, S.E. Sing, and S. Sriharan. 1994. Insecticidal activity of floral, foliar, and root extracts of Tagetes minuta (Asterales: Asteraceae) against adult Mexican bean weevils (Coleoptera: Bruchidae). Journal of Economic Entomology 87:1718-1725.

Wright, R.H. 1975. How mosquito repellents repel. Scientific American 233:104-111. 